\title{
Coevolution of economic and ecological systems
}

\section{An application to agricultural pesticide resistance}

\author{
Joëlle Noailly
}

Published online: 5 October 2007

(C) Springer-Verlag 2007

\begin{abstract}
This paper analyzes a model of economy-environment coevolution in which economic activities induce the genetic evolution of a biological species. This model is applied to the problem of pesticide resistance management. Just as in Munro (Environ Resour Econ, 9:429-449, 1997), we consider three main types of interactions: (1) a large pest population reduces economic revenues, (2) economic activities select for resistant genes and (3) the spread of resistant genes affects the size of the pest population. The model differs from Munro in that it includes evolutionary modeling of economic strategies. Economic agents are assumed to be boundedly rational, i.e they cannot compute the optimal level of pesticide use that minimizes resistance among pests. Economic evolution represents the change in the distribution of pesticide strategies in the population of economic agents and is modeled by a replicator dynamics equation. The interactions between economic evolution of pesticide strategies, pest population dynamics and genetic evolution of resistance of pests are studied in a system of three differential equations. We explore the dynamics and stability properties of the system using numerical simulations.
\end{abstract}

Keywords Evolution • Economics • Evolutionary modeling • Pesticide resistance $\cdot$ Antibiotic resistance

JEL Classification Q57 • B52 


\section{Introduction}

The concept of coevolution was first introduced in biology by Ehrlich and Raven (1964) to define the simultaneous reciprocal evolution of interacting populations. They observed that the patterns of evolution between plants and butterflies were closely interrelated. As plants developed toxic chemicals to prevent attacks from butterflies, the insects adapted by creating resistance to these chemicals. Examples of these patterns of coevolution abound in the biological world. Three main types of interactions are generally observed (Roughgarden 1979):

1. Parasitic relationships. In this case, one species benefits at the expense of the other. The butterflies-plants relationship described by Ehrlich and Raven (1964) is an example of a parasitic relationship.

2. Commensal relationships. One species benefits from another at negligible harm to the other species. An example is the presence of harmless bacteria living in human organisms (Hooper and Gordon 2001).

3. Mutualistic relationships. Both species benefit from one another. Fig trees, for instance, provide food for wasps, while the latter act as pollinators for the figs. As a result, the reproductive characteristics of the two species are intrinsically linked to one another (Herre 1989).

Models of coevolution in biology fuse population dynamics models with equations describing changes in gene frequencies. These models usually include a set of two differential equations for each interacting species. One equation represents population dynamics while the other reflects gene frequency changes (Levin and Udovic 1977; Roughgarden 1979; May and Anderson 1983). The idea behind this modeling approach is that numerical abundance of one species selects for the genetic characteristics of the other species, and conversely. Coevolutionary models are nowadays very sophisticated and include stochastic elements to describe sequences of mutations (Dieckmann et al. 1995; Dieckmann and Law 1996). Questions addressed by models of coevolution in biology relate to the final equilibrium configuration of ecological communities and the dynamics of coevolution, e.g. the presence of time lags in the adaptive response from one species to another. In recent years, the concept of coevolution has been transferred from the biological sphere to a large range of applications in socioeconomic contexts. For instance, the coevolution analogy has been used to describe the complex interactions between genes and culture (Lumsden and Wilson 1981), behavior and institutions (van den Bergh and Stagl 2003) and technology and industry (David and Rothwell 1996). In environmental economics, Norgaard (1984, 1994) was the first to suggest the use of coevolution to describe environmenteconomy interactions, although in a somewhat loose interpretation. In his view, development is shaped by a process of coevolution between several subsystems, namely, knowledge, values, organizations, technology, and the environment, so that each of these subsystems creates selective pressure on 
the others. As an illustration, Norgaard (1994) explains the current lock-in of fossil fuel technologies as the result of a coevolution of urbanization, technical progress and the development of the car industry.

Another example of environment-economy interaction is the coevolving pattern of human activity and climate change (Schneider and Londer 1984). By altering the composition of the atmosphere, human activity induces climate change, which forces society to adapt by way of the improved management of natural resources. Climate change in combination with intensive grazing, for instance, can cause desertification in certain areas, which, in turn, can force humans to migrate or to adopt cultures adapted to arid climates.

Using a strict definition, environment-economy coevolution refers to the fact that economic activities select for genetic traits of biological species. A well-studied example of such coevolution is the impact of fishing activities on the genetic evolution of fish (Stokes et al. 1993; Heino 1997). The choice of fishing gear and fishing mesh sizes selects for size and swimming abilities among the various species of fishes (Policansky 1993). In turn, these genetic changes force fishermen to adapt and modify their fishing strategies.

The objective of the present paper is to present a model of environmenteconomy coevolution, which reflects the impact of economic activities on the biological evolution of species, and vice versa. Using a coevolutionary framework may prove particularly useful in situations in which regulation is difficult, due for instance to the presence of bounded rationality. In these situations, environmental change cannot be perfectly anticipated, and economic activities are adaptive rather than optimal responses to this change. Although the concept of coevolution has been repeatedly mentioned in the literature, so far there has been little attempt explicitly to formalize it in a coevolutionary model. The present model of coevolution is applied to the problem of pesticide resistance management.

Pesticide resistance has become a subject of major concern. The National Research Council (1986) records that, between 1950 and 1980, nearly 500 pest species of agricultural importance have developed resistance to the principal classes of pesticides. This evolution, described in Fig. 1, is a direct consequence of the widespread use of intensive pesticide treatment in agriculture. Today, the spread of pesticide resistance poses major threats to agricultural yields. As an example, Tyler Miller (1992) states that, between 1940 and 1984, crop losses due to pests increased from 7 to $13 \%$, although there was a 12 -fold increase in pesticide use within the same period.

Economic models have treated the issue of pesticide resistance management in optimization models within the neoclassical economic tradition. Regev et al. (1983) derive the optimal dosage of pesticide under a constraint of decreasing pesticide susceptibility among pests, modeled as a non-renewable resource. This modeling choice eludes the issue of reversible resistance and is not based on a formal ecological theory. A similar approach of modeling resistance was used by Laxminarayan (2001) and Brown and Layton (1996) in dealing with antibiotic resistance. Munro (1997) offers a more satisfactory way to model 
Fig. 1 Increase in number of cases of resistant species (reproduced from American National Research Council (1986))

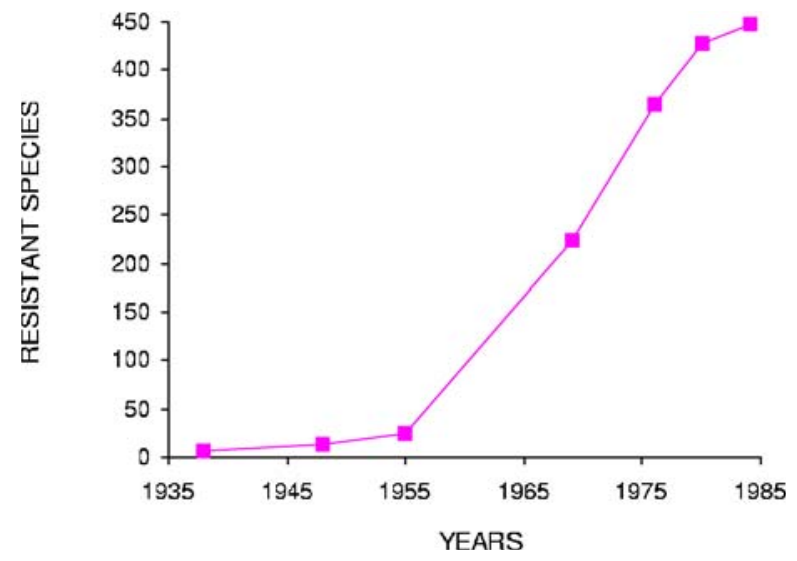

pest resistance using sound ecological-genetic modeling. The main intuition in his model is that economic activities reduce the fitness of genes, which confers susceptibility to pests, so that genes carrying resistant traits tend to be selected. Resistance allows the pest population to be less affected by pesticide use, so that the population of pests eventually recovers as more pests become resistant. Munro compares the optimal pesticide dosage of a myopic agent, who only understands pest population dynamics, but not genetic evolution, with that of a farsighted agent, who takes both pest population dynamics and genetic evolution into account when solving the optimization problem. He finds that the equilibrium obtained in the latter case exhibits a lower level of resistance and a lower pest population for the same level of pesticide use. Nevertheless, this model assumes perfect rationality of economic agents and is thus not a model of coevolution, since economic activities are not truly "evolving".

The model presented in this paper takes Munro's (1997) analysis as a starting point for the ecological side of the coevolutionary framework. Just as in Munro, we consider a situation in which a large area of land is used for the production of a crop. This crop is subject to a pest, whose presence reduces the yield of the crop. A population of individual farmers obtains revenues from harvesting the crop. The farmers use pesticides to limit pest nuisance and thus to increase crop yields and revenues. Nevertheless, intensive use of pesticides favors the development of resistant strains in the genetic structure of the pest. As resistance spreads, pesticides become less effective, and the pest population may eventually recover. In short, three main types of interactions must be considered: (1) a large pest population reduces economic revenues; (2) economic activities select for resistant genes; and (3) the spread of resistant genes affects the size of the pest population.

The model differs from Munro, however, in that it includes evolutionary modeling of economic strategies. Economic agents are assumed to be boundedly rational, i.e. they are not capable of anticipating any aspect of 
biological and ecological evolution and thus cannot compute the optimal level of pesticide use that minimizes resistance. Economic evolution represents the change in the distribution of pesticide strategies in the population of economic agents. In this model, economic and ecological evolution are combined in a system of differential equations. This makes the model truly "coevolutionary".

The analysis is organized as follows. Section 2 describes the model by presenting its three main building blocks: namely, genetic evolution of resistance; pest population dynamics; and economic evolution of agricultural pesticide strategies. Section 3 studies the steady states and discusses some general aspects of the dynamics of the coevolutionary system. Section 4 presents further results on possible forms of dynamic behavior using a numerical illustration. Section 5 draws policy implications from the analysis. Section 6 summarizes the main results and concludes.

\section{Description of the model}

\subsection{Selection of resistant genes}

This section models the evolution of genetic traits that make the pest either susceptible or resistant to pesticide use. The formalization of the ecological side of the model closely follows Munro's (1997) analysis. In turn, it is based on modeling in population genetics, a field of research in evolutionary biology concerned with the study of the collection of genes belonging to all members of a population, known as the "gene pool" (Roughgarden 1979).

We assume that pests affecting the crop are organisms whose reproduction is characterized by diploid inheritance. This means that the genetic structure of each pest is composed of pairs of chromosomes, one from each parent. Given matching chromosomes, we assume that there are only two types of genes: a gene $A$ that confers susceptibility to pests, and a gene $a$ that confers resistance. As a result, there are thus only three possible genotypes, or genetic structures, in the pest population: $A A, A a$ and $a a$.

Further, the $A$ gene is assumed to be dominant, implying that the presence of one $A$ gene in the genotype of a pest is sufficient to make the organism susceptible to pesticide. In contrast, the gene $a$ is recessive, meaning that the expression of resistance at the organism level requires the presence of $a$ genes on every chromosome. In other words, organisms of genotypes $A A$ and $A a$ are susceptible to pesticide use, while organisms of genotype $a a$ are resistant to it.

The fraction of genes $A$ and $a$ in the gene pool is denoted by $p$ and $q(=1-p)$, respectively. The fitness of an organism can be defined as the contribution which an individual makes to the next generation's gene pool, generally measured in number of offspring. For now, however, we ignore the effects of pesticide use on biological fitness. These will be introduced in Section 2.3. The fitness of a susceptible organism is $W_{A}$, while the fitness of a resistant organism is $W_{a}$. Given that, without pesticide use, $A$ genes are 
dominant, we have $W_{A}>W_{a}$. In addition, since each organism is composed of one pair of chromosomes, the proportion of genotype $A A$ in the gene pool is $p^{2}$, the proportion of genotype $A a$ is $2 p q$, and the proportion of genotype $a a$ is $q^{2}$. Obviously, we have $p^{2}+2 p q+q^{2}=1$. The average fitness over all genotypes in the gene pool is then:

$$
\begin{aligned}
\bar{W} & =p^{2} W_{A}+2 p q W_{A}+q^{2} W_{a} \\
& =p(2-p) W_{A}+q^{2} W_{a} .
\end{aligned}
$$

The dynamics of the proportion of susceptible pests $p$ can be described as follows:

$$
\dot{p}=\left(\frac{W_{A}}{\bar{W}}-1\right) p .
$$

This equation says that $p$ increases when the fitness of genes $A$ is above the average fitness.

\subsection{Dynamics of the size of the pest population}

This section formalizes the link between genetic evolution and the dynamics of the pest population. Following Munro (1997), we use tools from population ecology and evolutionary theory to model the fact that the evolution of the genetic distribution in the gene pool has an impact on pest population size.

Munro (1997) applies Roughgarden's (1979) analysis and assumes that the fitness of a gene is equal to: $(1+$ the individual's contribution to population growth). The individual's contribution to growth, or fitness, corresponds to the expected number of offspring produced by an individual. The formulation $(1+$ the individual's contribution to growth) reflects the fact that the number of offspring exceeds direct replacement. In addition, it is assumed that an individual's contribution to growth follows a logistic form. Fitness of genes is then:

$$
\begin{aligned}
& W_{A}=1+r_{A}\left(1-\frac{N}{K}\right) \\
& W_{a}=1+r_{a}\left(1-\frac{N}{K}\right),
\end{aligned}
$$

where $N$ is the size of the pest population, $r_{i}(i=A, a)$ is the contribution to growth $\left(r_{A}>r_{a}\right)$ in the absence of density effects, and $K>0$ is the (assumed) identical carrying capacity.

Pest population size grows with average fitness as follows:

$$
\dot{N}=(\bar{W}-1) N \text {. }
$$

Substituting for $W_{A}$ and $W_{a}$, defined by Eq. 3, into Eq. 1, average fitness becomes:

$$
\bar{W}=p^{2}\left(1+r_{A}-r_{A} \frac{N}{K}\right)+2 p q\left(1+r_{A}-r_{A} \frac{N}{K}\right)+q^{2}\left(1+r_{a}-r_{a} \frac{N}{K}\right) .
$$


Next, the evolution of pest population size can be obtained by substituting Eq. 5 into Eq. 4. We get:

$$
\begin{aligned}
\dot{N}= & p^{2} N\left(1+r_{A}-r_{A} \frac{N}{K}\right)+2 p q N\left(1+r_{A}-r_{A} \frac{N}{K}\right) \\
& +q^{2} N\left(1+r_{a}-r_{a} \frac{N}{K}\right)-N,
\end{aligned}
$$

which can be simplified as:

$$
\dot{N}=N\left[r-\frac{r N}{K}\right] .
$$

Here, $r$ is the average value of $r_{i},(i=A, a)$, with weighing as follows:

$$
r=p(2-p) r_{A}+q^{2} r_{a} .
$$

Equations 7 and 8 formalize the links between genetic evolution and population dynamics.

\subsection{Evolution of agricultural pesticide strategies}

In this section, the economic evolution of agricultural pesticide strategies is introduced into the model, using elements from the field of evolutionary economics. The modeling of the evolution of economic strategies is an innovative element. In contrast with Munro (1997), the evolutionary perspective is applied here not only to natural phenomena, but also to economic ones. This is motivated by the fact that, since agents are boundedly rational, they cannot calculate the optimal level of pesticide that maximizes their long-run discounted profits. Instead, economic agents adapt their decisions to changes occuring in the environment.

We consider a population of economic agents, or "farmers", composed of a fixed number $m$ of individuals. Farmers choose between two predetermined strategies: an "intensive" strategy that makes use of a high level of pesticides $x_{I}$, and a "biological" strategy that makes use of a low level of pesticides $x_{B}$ $\left(x_{I}>x_{B}\right){ }^{1}$ Obviously, additional strategies corresponding to a wider range of pesticide use could be implemented in this model, but, for simplicity, we limit our analysis to only two strategies.

In Munro (1997), individual revenues are a function of the pest population only. Here, we assume that revenues are affected by both the pest population size and individual's pesticide use. This corresponds to a more realistic

\footnotetext{
${ }^{1}$ In contrast with its common use in agriculture, the term "biological" does not necessarily refer here to a zero pesticide use strategy.
} 
situation in which the agents enjoy direct (short-run) benefits from the use of pesticides, as well as indirect (long-run) ones from the effects of total pesticide use on the pest population size. ${ }^{2}$ Further, we neglect the free-rider problem by assuming that the agents do not exhibit strategic behavior. In other words, they do not understand that increasing individual pesticide use when everyone else uses a biological strategy would generate larger profits. Another technical issue that motivates the introduction of individual pesticide use in the revenue function is that, if the revenue function were to depend only on $N$, the biological strategy would always yield larger profits than the intensive strategy, as $N$ is identical for all agents and a high level of pesticide use is more costly than a low level. In this case, the evolution of strategies can be easily predicted, as all agents would ultimately adopt the most profitable biological strategy. Individual profits are equal to net revenues of pesticide use:

$$
\pi_{j}=f\left(N, x_{j}\right)-c\left(x_{j}\right) \quad j=I, B .
$$

Here, $f\left(N, x_{j}\right)$ are the revenues from crop growth, and $c\left(x_{j}\right)$ represents the cost of pesticide use. In particular, we assume that $f_{N}<0$, i.e. the larger the pest population on the crop, the lower the individual revenues, and $f\left(N, x_{I}\right)>$ $f\left(N, x_{B}\right)$, i.e. large pesticide use generates large revenues. Further, we assume $c\left(x_{I}\right)>c\left(x_{B}\right)$, so that using a high level of pesticides is more costly than using a low level. Profits of farmers are not (directly) affected by the genetic evolution of resistance. In other words, $p$ does not enter the profit function. Therefore, farmers only need to have access to information about pest population size to calculate their profit levels.

Since the aggregate pest population affects all harvesters in the same way, an implicit assumption is that pests are equally distributed, i.e. can migrate, over the whole land area. The proportion of susceptible alleles in the gene pool of the pest population $(p)$ is assumed to be constant over all subpopulations, i.e. the level of resistance is the same on all land parcels.

The shares of farmers using the intensive and biological pesticide strategies are denoted by $s$ and $(1-s)$, respectively. The evolution of pesticide strategies shares is described using a replicator dynamic equation:

$$
\dot{s}=s\left(\pi_{I}-\bar{\pi}\right),
$$

where $\bar{\pi}$ denotes average profits, such that $\bar{\pi}=s \pi_{I}+(1-s) \pi_{B}$. The replicator dynamic equation reflects the fact that agents experience (imperfect or delayed) social learning, and update strategies by imitating the strategy that yields above-average profits. Indeed, the share of the intensive pesticide strategy grows as long as $\pi_{I}>\bar{\pi}$. Further on, we will adopt the following

\footnotetext{
${ }^{2}$ Another interpretation, which could be clarified in a spatial model, is that individual revenues are affected by both local effects, through individual pesticide use, and global effects, through the aggregate pest population size.
}

Springer 
functional forms that capture the aforementioned assumptions on revenues and cost functions:

$$
\begin{aligned}
f\left(N, x_{j}\right) & =\frac{a x_{j}}{N+1}, \quad j=I, B, \\
c\left(x_{j}\right) & =b x_{j}^{2},
\end{aligned}
$$

so that Eq. 10 is equivalent to:

$$
\dot{s}=s(1-s)\left(x_{I}-x_{B}\right)\left(\frac{a}{N+1}-b\left(x_{I}+x_{B}\right)\right) .
$$

Economic activities reduce the fitness of susceptible genes as follows:

$$
W_{A x}=W_{A}-m\left(s x_{I}+(1-s) x_{B}\right) .
$$

Here, $W_{A x}$ corresponds to the reduced fitness of genes $\mathrm{A}$ as a result of total pesticide use. If human activities reduce the fitness of susceptible genes, such that $W_{A x}<W_{a}$, then resistant strains will be selected over susceptible ones. The average fitness, given pesticide use denoted by $\bar{W}_{x}$, is defined by:

$$
\bar{W}_{x}=\bar{W}-p(2-p) m\left(s x_{I}+(1-s) x_{B}\right) .
$$

The evolution of $p$ over time is then:

$$
\dot{p}=\left(\frac{W_{A x}}{\bar{W}_{x}}-1\right) p .
$$

Substituting Eqs. 13, 14 and 1 in Eq. 15, and rewriting gives:

$$
\dot{p}=\frac{p q^{2}\left(W_{A}-m\left(s x_{I}+(1-s) x_{B}\right)-W_{a}\right)}{p(2-p)\left(W_{A}-m\left(s x_{I}+(1-s) x_{B}\right)\right)+q^{2} W_{a}} .
$$

Further, substituting for Eqs. 3 and 13, where $W_{A}$ is replaced by $W_{A x}$, into Eq. 1 gives another expression for $\bar{W}_{x}$ :

$$
\begin{aligned}
\bar{W}_{x}= & p^{2}\left(1+r_{A}-r_{A} \frac{N}{K}-m\left(s x_{I}+(1-s) x_{B}\right)\right) \\
& +2 p q\left(1+r_{A}-r_{A} \frac{N}{K}-m\left(s x_{I}+(1-s) x_{B}\right)\right) \\
& +q^{2}\left(1+r_{a}-r_{a} \frac{N}{K}\right) .
\end{aligned}
$$

The evolution of pest population size can be obtained by substituting Eq. 17 into Eq. 4 , where $\bar{W}$ is replaced by $\bar{W}_{x}$. After rewriting, we obtain:

$$
\dot{N}=N\left[\left(p(2-p) r_{A}+q^{2} r_{a}\right)\left(1-\frac{N}{K}\right)-p(2-p) m\left(s x_{I}+(1-s) x_{B}\right)\right] \text {. }
$$




\section{Dynamic analysis of the coevolutionary system}

\subsection{The coevolutionary system}

The full model of coevolution is represented by a system of three differential equations: namely, Eqs. 12, 16 and 18.

$$
\left\{\begin{array}{l}
\dot{s}=s(1-s)\left(x_{I}-x_{B}\right)\left(\frac{a}{N+1}-b\left(x_{I}+x_{B}\right)\right) \\
\dot{N}=N\left[\left(p(2-p) r_{A}+q^{2} r_{a}\right)\left(1-\frac{N}{K}\right)-p(2-p) m\left(s x_{I}+(1-s) x_{B}\right)\right] \\
\dot{p}=\frac{p q^{2}\left(W_{A}-m\left(s x_{I}+(1-s) x_{B}\right)-W_{a}\right)}{p(2-p)\left(W_{A}-m\left(s x_{I}+(1-s) x_{B}\right)\right)+q^{2} W_{a}}
\end{array}\right.
$$

with $0 \leq s \leq 1,0 \leq N \leq K$, and $0 \leq p \leq 1$ and $q=1-p$.

The system includes two equations for the ecological part of the system and one equation for the economic system. Note that a full model of coevolution, analogous to the ones developed in biology, would require two equations for each system, one for the genetic-strategy evolution and one for the population dynamics. In the economic context, this would require the relaxation of the assumption of a fixed population of farmers, for instance, by allowing for entry and exit of agents. Nevertheless, in the present model, we assume that the population of harvesters is fixed. This simplifies the model greatly, allowing for a first discussion of coevolutionary systems.

\subsection{Identification of the steady states}

The steady states of the dynamic system are situated at the intersections of the nullclides $\dot{N}=0, \dot{p}=0$ and $\dot{s}=0$. We find $\dot{s}=0$ along the two-dimensional planes defined by $s=0, s=1$ and $N=\bar{N}$, where $\bar{N}$ is given by:

$$
\bar{N}=\frac{a}{b\left(x_{I}+x_{B}\right)}-1 .
$$

Next, we find $\dot{N}=0$ along the planes $N=0$ and $s=g(N, p)$, as given by:

$$
s=g(N, p)=\frac{\left(p(2-p) r_{A}+q^{2} r_{a}\right)(K-N)-x_{B} \operatorname{Kmp}(2-p)}{K m p(2-p)\left(x_{I}-x_{B}\right)} .
$$

Finally, we have $\dot{p}=0$ along the planes $p=0, p=1$ and $s=\bar{s}$, where $\bar{s}$ is given by:

$$
\bar{s}=\frac{W_{A}-W_{a}-m x_{B}}{m\left(x_{I}-x_{B}\right)} .
$$

The details of solving the system and the existence conditions of each steady state are given in Appendices 1 and 2, respectively. 
In total, ten steady states $\left(\mathrm{A}-\mathrm{F}\right.$ and $\left.\mathrm{O}_{1}-\mathrm{O}_{4}\right)$ can be identified:
A. $\left(N_{A} ; p_{A} ; s_{A}\right)=\left(\frac{K r_{A}-x_{I} K m}{r_{A}} ; 1 ; 1\right)$.
B. $\left(N_{B} ; p_{B} ; s_{B}\right)=\left(\frac{K r_{A}-x_{B} K m}{r_{A}} ; 1 ; 0\right)$.

The steady states A and B are characterized by the absence of resistance. At these points $p=1$, so that there are only susceptible genes in the gene pool. At A, all agents use the intensive strategy $x_{I}$, so that the pest population is lower than in the steady state $\mathrm{B}$, in which all agents use the low level of pesticide $x_{B}$.

C. $\left(N_{C} ; p_{C} ; s_{C}\right)=(K ; 0 ; 1)$.

D. $\left(N_{D} ; p_{D} ; s_{D}\right)=(K ; 0 ; 0)$.

The steady states $\mathrm{C}$ and $\mathrm{D}$ are characterized by the sole presence of resistant genes, since $p=0$. At these points, the population of pests reaches the carrying capacity level $K$. Just as for A and B, there is one steady state (C) where all agents use the intensive strategy, and another (D) where all agents use the biological strategy.

E. $\left(N_{E} ; p_{E} ; s_{E}\right)=\left(\bar{N} ; 1-\sqrt{\frac{v}{u}} ; \bar{s}\right)$,

where $u$ and $v$ are analytical expressions defined by Eqs. 29 and 30 given in Appendix 1. The steady state $\mathrm{E}$ is characterized by a pest population size that falls between $N_{A}$ and $N_{C}$, an intermediate level of resistance, and the coexistence of both intensive and biological pesticide strategies in the economic sphere. At the steady state E, both strategies yield equal profits.

F. $\left(N_{F} ; p_{F} ; s_{F}\right)=\left(\bar{N} ; 1 ; \frac{r_{A}\left(b\left(x_{I}+x_{B}(K+1)-a\right)-x_{B} b K m\left(x_{I}+x_{B}\right)\right.}{b K m\left(x_{I}^{2}-x_{B}^{2}\right)}\right)$.

The steady state $\mathrm{F}$ is characterized by a pest population size of $\bar{N}$, no resistance $\left(p_{F}=1\right)$, and the coexistence of both pesticide strategies. At F, profits yielded by both strategies are equal (and are also equal to profits obtained at $\mathrm{E}$ ).

$\mathrm{O}_{1} .(0 ; 0 ; 0 ;)$.

$\mathrm{O}_{2} .(0 ; 0 ; 1)$.

$\mathrm{O}_{3} .(0 ; 1 ; 0)$.

$\mathrm{O}_{4} \cdot(0 ; 1 ; 1)$.

Finally, we can identify four equilibria which correspond to extreme cases where the pest population is extinct.

Figure 2 represents the nullclides and the steady states of the system in the three-dimensional space in the particular case in which all steady states lie in the feasible range for $N, s$ and $p$, i.e. $0 \leq N \leq K, 0 \leq s \leq 1$ and $0 \leq p \leq 1$.

\subsection{Dynamics}

The direction of motion in the three-dimensional space is given by:

- $\quad \dot{s}>0$ for $0<s<1$ and $N<\bar{N}$. The threshold $\bar{N}$ is the level of pest population, below (above) which the proportion of agents using the intensive 
Fig. 2 Graphical representation of the system in three-dimensional space

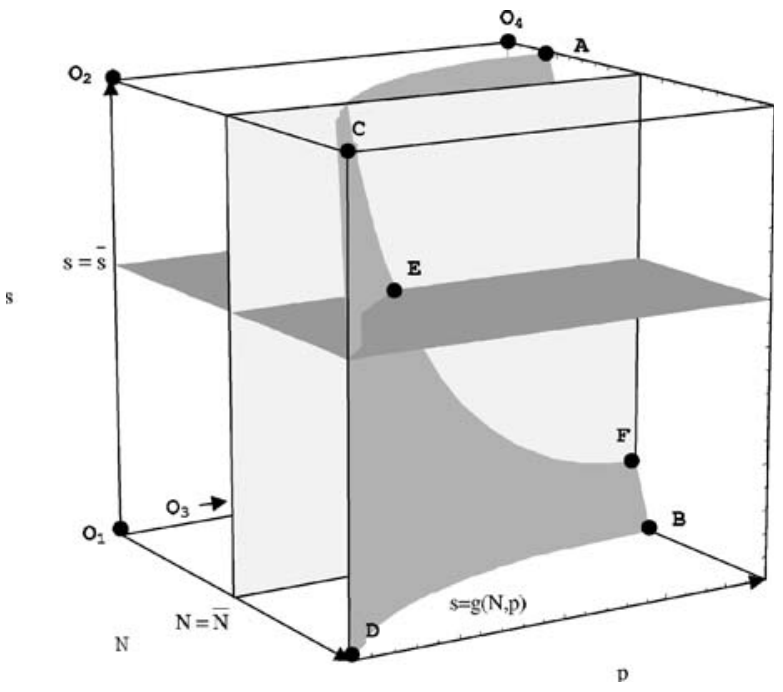

strategy increases (decreases). Indeed, when the pest population is lower than $\bar{N}$, the intensive strategy yields larger profits than the biological strategy, and an increasing number of agents adopt the intensive stategy. At $N=\bar{N}$, the strategies coexist, as they yield equal positive profits $\pi_{I}=$ $\pi_{B}=b x_{I} x_{B}$, and the evolution of strategies stops.

- $\quad \dot{N}>0$ for $N<K$ and $s<g(N, p)$. When the proportion of agents using the intensive strategy lies below (above) the locus $s=g(N, p)$, the pest population increases (decreases). Intuitively, the pest population is negatively affected by a large proportion of susceptible genes $p$ combined with a large pesticide use. Indeed, when resistance is low in the system, pesticides are efficient in killing pests. If, in addition, many agents use the intensive strategy, $N$ will decrease sharply. Therefore, a large $p$ and a large $s$ cause $N$ to decrease rapidly. However, when $p$ is small and almost all pests are resistant, pesticides are not efficient in killing pests, and then the population of pests increases for any level of pesticide use in the population, i.e. for any level of $s$.

- $\quad \dot{p}>0$ for $0<p<1$ and $s<\bar{s}$. The threshold $\bar{s}$ corresponds to the proportion of intensive strategies, above (below) which resistance increases (decreases) in the system. Whenever the proportion of intensive strategies is larger than $\bar{s}$, then $W_{A x}<W_{a}$. Hence, resistant genes increase in proportion in the gene pool and $p$ decreases. At $\bar{s}$, the evolution of resistance is halted.

Due to the high dimensionality of the system, analytical tractability is lost. Therefore, the remainder of the analysis is conducted using numerical experiments. Given the large number of parameters in the system, simulations are carried out for a fixed range of parameters providing exploratory results. The objective is thus limited to providing some intuition regarding the long-run behavior of such environment-economy coevolution systems. 


\section{A numerical illustration of the coevolutionary system}

\subsection{Parameter values and steady states}

Numerical simulations are carried out to illustrate the dynamical behavior of the system. The numerical experiments were implemented in Mathematica and checked using an ODE solver for stiff equations in Matlab. We fix the parameters as follows:

$$
\begin{array}{rlrl}
x_{I} & =0.05 & x_{B} & =0.025 \\
K & =100 & m & =10 \\
a & =20 & b & =5 \\
r_{A} & =0.6 & r_{a} & =0.4 \\
W_{A} & =1 & W_{a} & =0.6 .
\end{array}
$$

The choice of parameter values for ecological parameters $r_{A}, r_{a}, W_{a}$ and $W_{A}$ is based on numerical examples in Roughgarden (1979). The fitness levels $W_{A}$ and $W_{a}$ can be interpreted as an individual's contribution to the next generation in terms of a (scaled) number of offspring. Pesticide use parameters measure a quantity of pesticide use, but can be, in the present context, broadly interpreted as a measure of the (scaled) number of offspring killed. Further, the size of the system is small: we consider only 10 farmers and a maximum pest population of $K=100$. This parameter choice ensures that all steady states A, $\mathrm{B}, \mathrm{C}, \mathrm{D}, \mathrm{E}, \mathrm{F}, \mathrm{O}_{1}, \mathrm{O}_{2}, \mathrm{O}_{3}$ and $\mathrm{O}_{4}$ exist. The coordinates of the steady states are then:
A. $\left(N_{A} ; p_{A} ; s_{A}\right)=(16.66 ; 1 ; 1)$.
B. $\left(N_{B} ; p_{B} ; s_{B}\right)=(58.33 ; 1 ; 0)$.
C. $\left(N_{C} ; p_{C} ; s_{C}\right)=(100 ; 0 ; 1)$.
D. $\left(N_{D} ; p_{D} ; s_{D}\right)=(100 ; 0 ; 0)$.
E. $\left(N_{E} ; p_{E} ; s_{E}\right)=(52.33 ; 0.388 ; 0.6)$.
F. $\left(N_{F} ; p_{F} ; s_{F}\right)=(52.33 ; 1 ; 0.144)$.
$\mathrm{O}_{1} .(0 ; 0 ; 0)$.
$\mathrm{O}_{2} .(0 ; 0 ; 1)$.
$\mathrm{O}_{3} .(0 ; 1 ; 0)$.
$\mathrm{O}_{4} \cdot(0 ; 1 ; 1)$.

To avoid cumulative errors of approximation close to $p=0$ leading to the possible occurrence of negative values for $p$, the simulations were carried out using a logarithmic transformation of $p .^{3}$ All simulations were run over 50,000 time steps which appeared to be long enough to observe asymptotic convergence to a steady state.

\footnotetext{
${ }^{3}$ We computed the simulations using a transformed system formed by Eqs. 19, 20, in which $p$ is replaced by $e^{p}$. Further, Eq. 21 is transformed into:

$$
(\ln p)=\frac{\left(1-e^{p}\right)^{2}\left(W_{A}-m\left(s x_{I}+(1-s) x_{B}\right)-W_{a}\right)}{e^{p}\left(2-e^{p}\right)\left(W_{A}-m\left(s x_{I}+(1-s) x_{B}\right)\right)+\left(1-e^{p}\right)^{2} W_{a}} .
$$


Fig. 3 Coevolution of $N, s$ and $p$, with $N_{0}=K, p_{0}=0.8$, and $s_{0}=0.25$

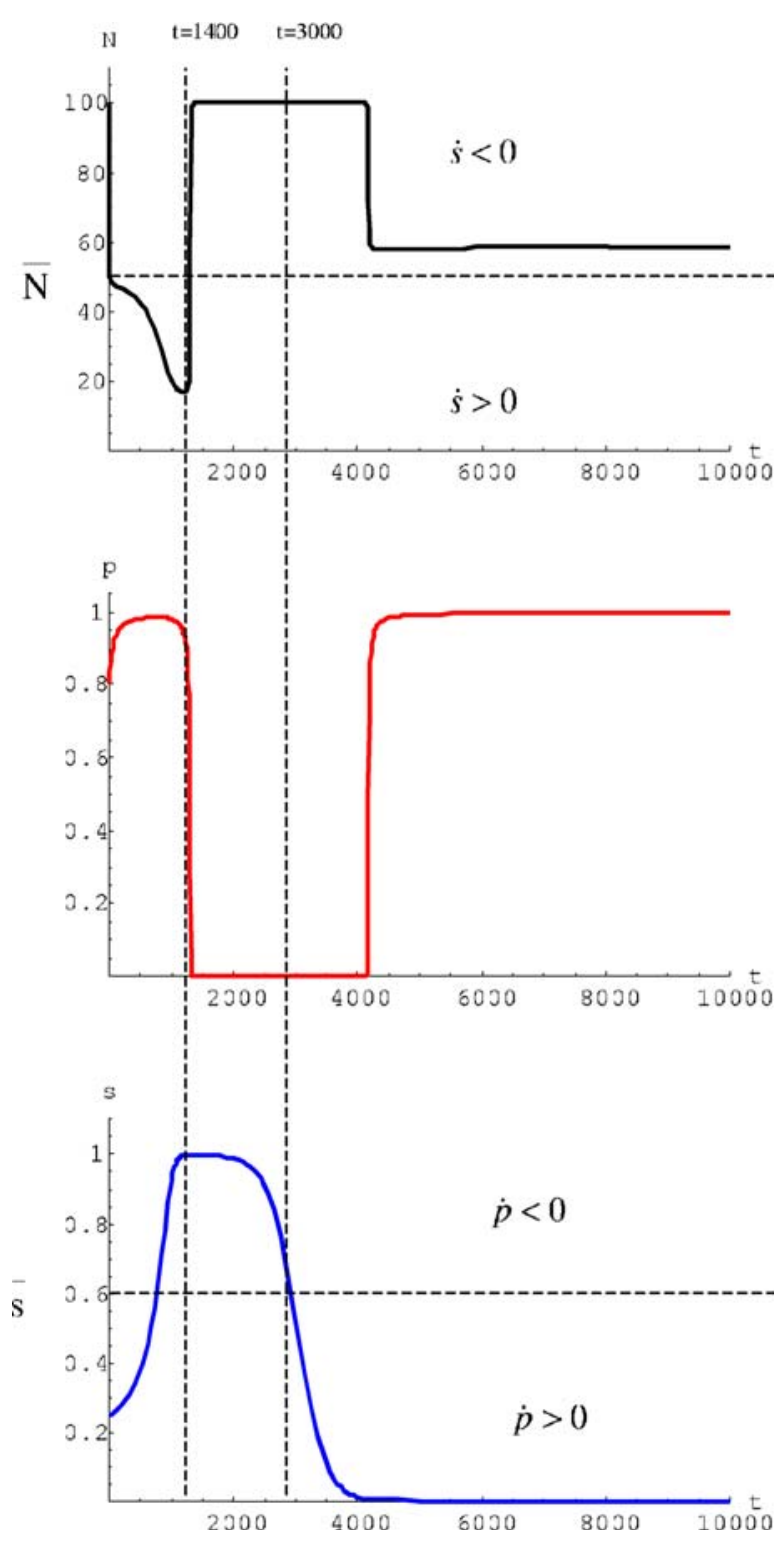

As an illustration, Fig. 3 presents the behavior of the system, starting with the following initial conditions: $N_{0}=K, s_{0}=0.25$ and $p_{0}=0.8$.

Under the chosen parameters, the coevolutionary system converges to the steady state B. The intuition behind the simultaneous evolution of $N, p$ and $s$ is as follows:

1. Between $t=0$ and $t \simeq 1400$. The pest population decreases sharply, since $p$ is initially large, and the pest population is negatively affected by pesticide use. This can be observed in the upper graph of Fig. 3. This large drop in 
$N$ occurs within the first 60 time steps. ${ }^{4}$ As soon as the size of the pest population falls below $\bar{N}, s$ starts to increase progressively. Since there are few farmers using the intensive strategy initially $\left(s_{0}<\bar{s}\right)$, no resistance is created and the proportion of susceptible genes increases. This occurs until $t \simeq 750$. As $s$ keeps on increasing and passes above $\bar{s}$, resistance is created in the system and $p$ decreases, first gradually and then sharply. As $p$ decreases, pesticides become less efficient in killing pests and the pest population recovers. At $t \simeq 1400, p$ gets very close to zero (without ever reaching it ${ }^{5}$ ), while $N$ reaches the carrying capacity.

2. Between $t \simeq 1400$ and $t \simeq 3000$. At about $t \simeq 1400, N$ exceeds $\bar{N}$ and the intensive strategy yields less than average profits. As a result, $s$ decreases progressively.

3. At $t \simeq 3000$. As $s$ passes below $\bar{s}$, pesticides no longer create resistance and $p$ rises (see the logarithmic graph of $p$ in Fig. 8 in Appendix 3). As $p$ increases, pesticides become efficient in killing pests and $N$ falls sharply. Nevertheless, $N$ does not fall below $\bar{N}$. Indeed, as $s$ is then close to zero, the drop in $N$ is stopped and the pest population size converges to $N_{B}$. In the long-run, $s$ converges asymptotically to zero, as $\pi_{B}$ remains larger than $\pi_{I}$. Only susceptible genes are left in the gene pool and $p$ asymptotically approaches 1 .

Figure 4 shows the three-dimensional phase diagram of the system. ${ }^{6}$ Figure 5 describes the evolution of the profit differential $\left(\pi_{I}-\pi_{B}\right)$ over time. This can be analyzed in parallel with the evolution of $N$ and $s$ in the upper and lower graph of Fig. 3. First, we observe a positive profit differential, implying that $\pi_{I}>\pi_{B}$ until about $t \simeq 1400$. This corresponds to $N$ smaller than $\bar{N}$ in the upper graph of Fig. 3, and to the gradual increase of $s$ in the lower graph of Fig. 3. The ranking of profits switches at around $t \simeq 1400$ and, after this point, the profit differential remains negative, i.e. $\pi_{B}$ remains larger than $\pi_{I}$ and $s$ converges to zero.

The speed at which each variable evolves deserves further explanation.

- The evolution of $N$ depends on two forces: (1) the natural growth rate of the pest population $r N\left(1-\frac{N}{K}\right)$ that augments the pest population, and (2) the killing of pests by pesticide use, which is a function of $p$ and $s$, that reduces it. The rate at which pests are killed depends on the level of resistance in the population. In other words, when $p$ is large (small), the number of pests killed is large (small). The quick drops and rises in $N$ are fully explained by the characteristics of the logistic equation. Indeed, where $N$ is near $K$, the natural growth rate of the population is close to

\footnotetext{
${ }^{4}$ This short-run drop cannot be well observed in Fig. 3 due to the presentation of coevolutionary dynamics on a long-run horizon.

${ }^{5}$ The dynamic behavior of $p$ close to $p=0$ is best observed using the logarithmic transformation as shown in Fig. 8 in Appendix 3.

${ }^{6}$ For illustration purposes, the axes of $N$ and $p$ are reversed.
} 
Fig. 4 Three-dimensional phase diagram, with $N_{0}=100, p_{0}=0.8$, and $s_{0}=0.25$

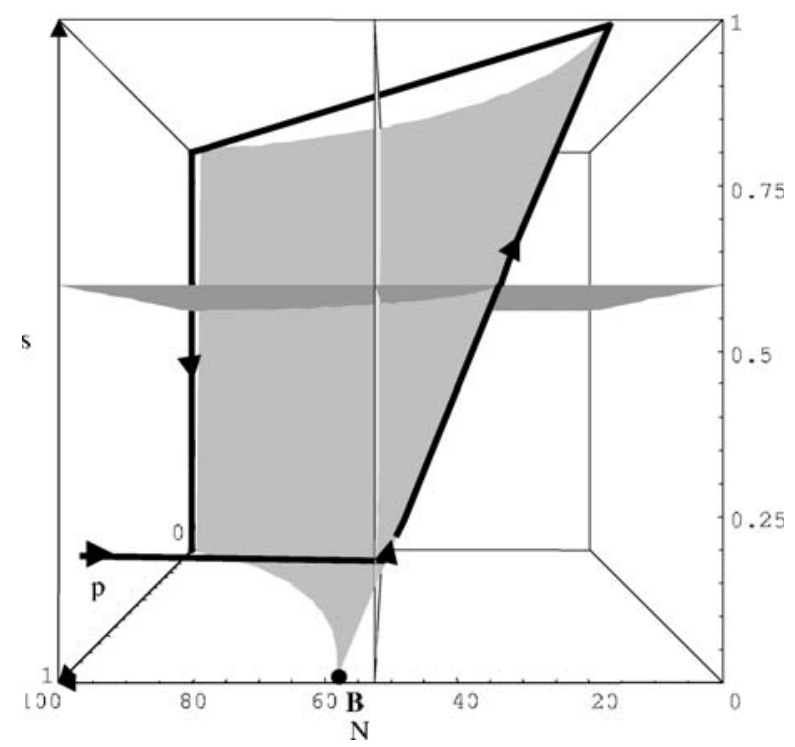

zero. When, at the same time, $p$ is large, $N$ will decrease sharply since the killing of pests then far exceeds the natural growth rate. This explains the two sharp declines in $N$ observed just after $t=0$ and $t \simeq 3,500$. At both points, $p$ is large and $N$ is near $K$. But, when $N$ is much smaller than $K$, then the natural growth rate of the pest population is close to $r N$, i.e. it exhibits exponential growth, implying that the pest population replenishes quickly. When, at the same time, resistance is large ( $p$ close to zero), then the killing of pests by pesticide use is relatively low and the replenishment of pests occurs faster than their killing. This explains the sharp rise in $N$ observed around $t \simeq 1,400$. Finally, between $t \simeq 60$ and $t \simeq 1,400$, the slow decrease in $N$ is caused by the fact that the killing of pests is just slightly larger than their natural growth rate. After $t \simeq 4,000$, on the path

Fig. 5 Evolution of profits differential over time, with $N_{0}=100, p_{0}=0.8$, and $s_{0}=0.25$

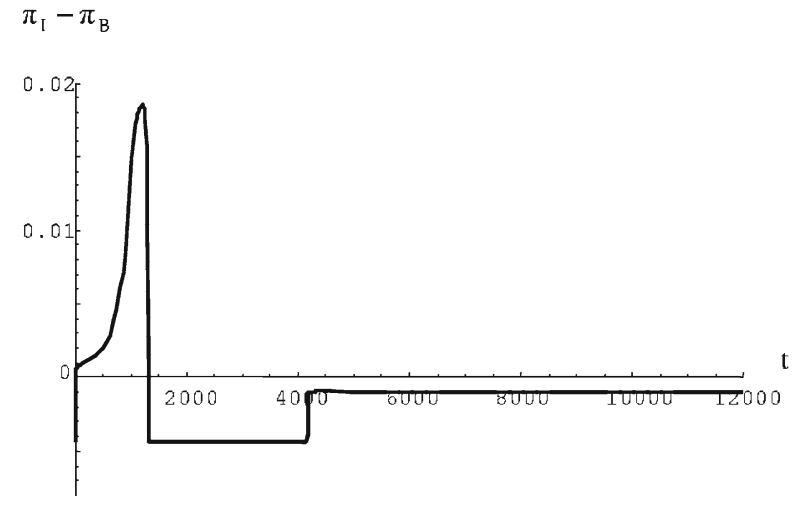


converging to the steady state $\mathrm{B}$, the natural growth rate almost exactly compensates the killing of pests, and, in the long run, $N$ approaches the equilibrium level $N_{B}$.

- The evolution of $p$ depends on the proportion of $A$ genes present in the population. As observed in the middle graph of Fig. 3, after $t \simeq 750$, the decline in $p$ due to large pesticide use starts out rather flat but accelerates as evolution progresses. This is because, at the beginning, the $a$ a phenotype is very rare and only a very small fraction of the population is being favored by selection, all the rest being selected against. Nevertheless, as evolution proceeds, more and more $a$ genes are present in the gene pool and the speed increases until $p$ gets close to zero. The same mechanism explains the increase in $p$ observed around $t \simeq 3,500$. At that point, $A$ genes start to be selected over $a$ ones. As $A$ genes are rare, evolution starts slowly but accelerates sharply as these become more frequent in the gene pool.

- In comparison with $N$ and $p$, the economic evolution of strategies follows a gradual process. This is due to the specific functional form of the replicator dynamics used. Indeed, the evolution of $s$ is a function of $s(1-s)$, and of the profit differential between $\pi_{I}$ and $\bar{\pi}$. The rate of change in $s$ therefore depends on the proportion of intensive strategies, as well as on the proportion of biological ones. The role of $(1-s)$ explains that economic evolution does not exhibit patterns of acceleration, as observed for $p$. For instance, when $s$ is very small and $\pi_{I}>\pi_{B}, s$ will only increase gradually. A possible interpretation of this gradual change is that, when many agents use the biological strategy, there is a relatively low probability of observing an intensive agent earning larger profits. In other words, intensive agents are more difficult to detect in a population composed mainly of biological farmers. This can be explained by the fact that agents are boundedly-rational and only observe a limited set of agents, most likely in their immediate neighborhood. Therefore, the speed of imitation of the most profitable strategy will be rather slow.

In this illustration, economic and ecological phenomena evolve on the same time-scale. Biological studies on pest resistance have shown that agricultural pests and bacteria may exhibit very short reproductive cycles, thus leading to biological evolution occuring very rapidly. Certain species of flies, for instance, have evolved important resistance to insecticides within time scales of one or two years. The potato wireworm evolved large-scale resistance after only 5 years of exposure (Georghiou 1980). Nevertheless, in most of the cases, biological evolution will occur over much longer time scales than economic evolution. Decoupling the time scales of economic and biological evolution can be easily introduced in this model. For instance, multiplying the right-hand side of Eq. 19 by a time-scale parameter would make it possible to control for the speed of economic evolution. A large parameter would then imply that economic evolution occurs much faster than biological evolution. A simpler way to speed up economic evolution in comparison to biological evolution is to decrease the parameter $x_{B}$, so as to increase the difference $\left(x_{I}-x_{B}\right)$ in Eq. 19 . 


\subsection{Stability properties of the steady states}

\subsubsection{Local stability results: linearization}

In this section, we study the local stability properties of each steady state by looking at the properties of the linearized system near the steady states, using Jacobian analysis. This provides only partial information about the long-run behavior of the system, but makes it possible to rule out stability for certain steady states.

We evaluate numerically the eigenvalues of the Jacobian matrix with the same set of parameters given above. The signs of the respective eigenvalues of the Jacobian matrix and the resulting stability properties are given in Table 1.

The steady state $\mathrm{E}$ has one real eigenvalue and two eigenvalues that form complex conjugates. All other equilibria exhibit only real eigenvalues. The local stability theorem (Strogatz 1994; Hilborn 1994) states that the steady state is locally asymptotically stable if all the eigenvalues of the respective Jacobian matrix have negative real parts, or if at least two eigenvalues are negative and one is zero. Local stability can thus be concluded for A and B, but not for $\mathrm{F}$ and $\mathrm{O}_{4}$. Along the same lines, the steady state is unstable if all eigenvalues are positive, or at least two are positive and one is zero. Therefore, we can rule out stability for the steady states $\mathrm{O}_{1}, F$ and $\mathrm{O}_{4}$.

Next, eigenvalues of opposite signs indicate that the steady state is a saddle point. This is the case for the steady states $\mathrm{C}, \mathrm{D}, \mathrm{E}$ and $\mathrm{O}_{2}$. A saddle point indicates that the stability of the steady state is conditional on initial conditions. There exists a trajectory, such that this state can be reached. However, for arbitrary initial conditions, this will only occur by chance.

Table 1 Local stability results

\begin{tabular}{|c|c|c|c|c|c|}
\hline \multirow[t]{2}{*}{ Steady states } & \multicolumn{3}{|c|}{ Signs of eigenvalues } & \multirow[t]{2}{*}{ Singular points } & \multirow[t]{2}{*}{ Stability result } \\
\hline & $\lambda_{1}$ & $\lambda_{2}$ & $\lambda_{3}$ & & \\
\hline A & - & - & 0 & Node & Locally stable \\
\hline B & - & - & 0 & Node & Locally stable \\
\hline $\mathrm{C}$ & - & - & + & Saddle point & $\begin{array}{l}\text { Stability depends } \\
\text { on initial conditions }\end{array}$ \\
\hline $\mathrm{D}$ & - & + & - & Saddle point & $\begin{array}{l}\text { Stability depends on } \\
\text { initial conditions }\end{array}$ \\
\hline $\mathrm{E}$ & - & $\operatorname{Re}\left(\lambda_{2}\right)>0$ & $\operatorname{Re}\left(\lambda_{3}\right)>0$ & Saddle point & $\begin{array}{l}\text { Stability depends on } \\
\text { initial conditions }\end{array}$ \\
\hline $\mathrm{F}$ & - & + & 0 & & $\begin{array}{c}\text { Cannot be determined } \\
\text { by Jacobian analysis }\end{array}$ \\
\hline $\mathrm{O}_{1}$ & + & + & + & Repellor & Unstable \\
\hline $\mathrm{O}_{2}$ & - & + & - & Saddle point & $\begin{array}{l}\text { Stability depends on } \\
\text { initial conditions }\end{array}$ \\
\hline $\mathrm{O}_{3}$ & + & + & 0 & Repellor & Unstable \\
\hline $\mathrm{O}_{4}$ & - & + & 0 & & $\begin{array}{c}\text { Cannot be determined } \\
\text { by Jacobian analysis }\end{array}$ \\
\hline
\end{tabular}




\subsubsection{Global stability results: sensitivity analysis}

To determine under which conditions the system converges (or not) to each steady state, we run a sensitivity analysis for a wide range of initial conditions. We let $N_{0}$ vary between 5 and 100 in increasing steps of 5 . Further, we let $s_{0}$ and $p_{0}$ vary between 0.05 and 0.95 in increasing steps of 0.05 .

Some straightforward canonical cases can be briefly studied here. For $s_{0}=0$ and under the chosen parameters, the coevolutionary system (Eqs. 19-21) will converge to the steady state B. Next, for $s_{0}=1$, the system converges to C. Further, starting with $p_{0}=0$, the system reaches D. Finally, for $N_{0}=0$ the system converges to $\mathrm{O}_{2} .7$

Only two types of steady state emerge out of the numerical experiments, namely, B and C. B corresponds to a situation in which all agents use the biological strategy, there is no resistance in the system, and the pest population levels out at a large level, but remains below the carrying capacity. At B, boundedly rational economic agents keep on using the biological strategy, although pests could be efficiently killed by using larger levels of pesticides. This behavior, however, make sense from an economic perspective, since using larger amounts of pesticides, i.e. using an intensive strategy, is very costly, as long as $N$ is larger than $\bar{N}$. At $\mathrm{B}$, the biological strategy remains more profitable than the intensive one, so farmers have no incentive to change strategy.

The state C corresponds to a 'degenerate' steady state, in which all farmers use the costly intensive pesticide strategy even though pesticides are ineffective, since all pests are resistant $(p=0)$. In our simulations, convergence to $\mathrm{C}$ is observed in $7 \%$ of the simulation runs. Initial conditions leading to $\mathrm{C}$ are irregularly scattered over the whole map of initial values. An example of convergence to $\mathrm{C}$ is presented in Fig. $6 .^{8}$

Convergence to $\mathrm{C}$ is caused by the fact that, under certain circumstances, the adaptive response of ecological evolution is characterized by long delays. In Fig. $6, N$ remains below $\bar{N}$ for a long period of time, between about $t \simeq 4,000$ and $t \simeq 9,000$. As a result, the share of intensive pesticide strategies increases and eventually $s$ reaches 1 . As $s$ passes above $\bar{s}, p$ starts to decrease. Nevertheless, there exists a time lag between the evolution of strategies and the consequence of this evolution on resistance. In Fig. 6, the sharp decrease in $p$, causing $N$ to jump to $K$, occurs too late to reverse economic evolution. In fact, all farmers have already switched to the intensive strategy before the effects on the ecological system become too important. In this case, since all

\footnotetext{
${ }^{7}$ The boundary cases $N_{0}=K$ and $p_{0}=1$ cannot be determined in a straightforward way. We exclude the canonical cases $s=0, s=1, p=0, p=1$ from our simulations, since these imply that both economic and genetic evolution are halted. Instead, we are interested in truly coevolutionary dynamics.

${ }^{8}$ See the logarithmic graph of $p$ in Fig. 9 in Appendix 3.
} 
Fig. 6 Convergence to $\mathrm{C}$ with initial conditions $N_{0}=50$, $p_{0}=0.5$, and $s_{0}=0.8$
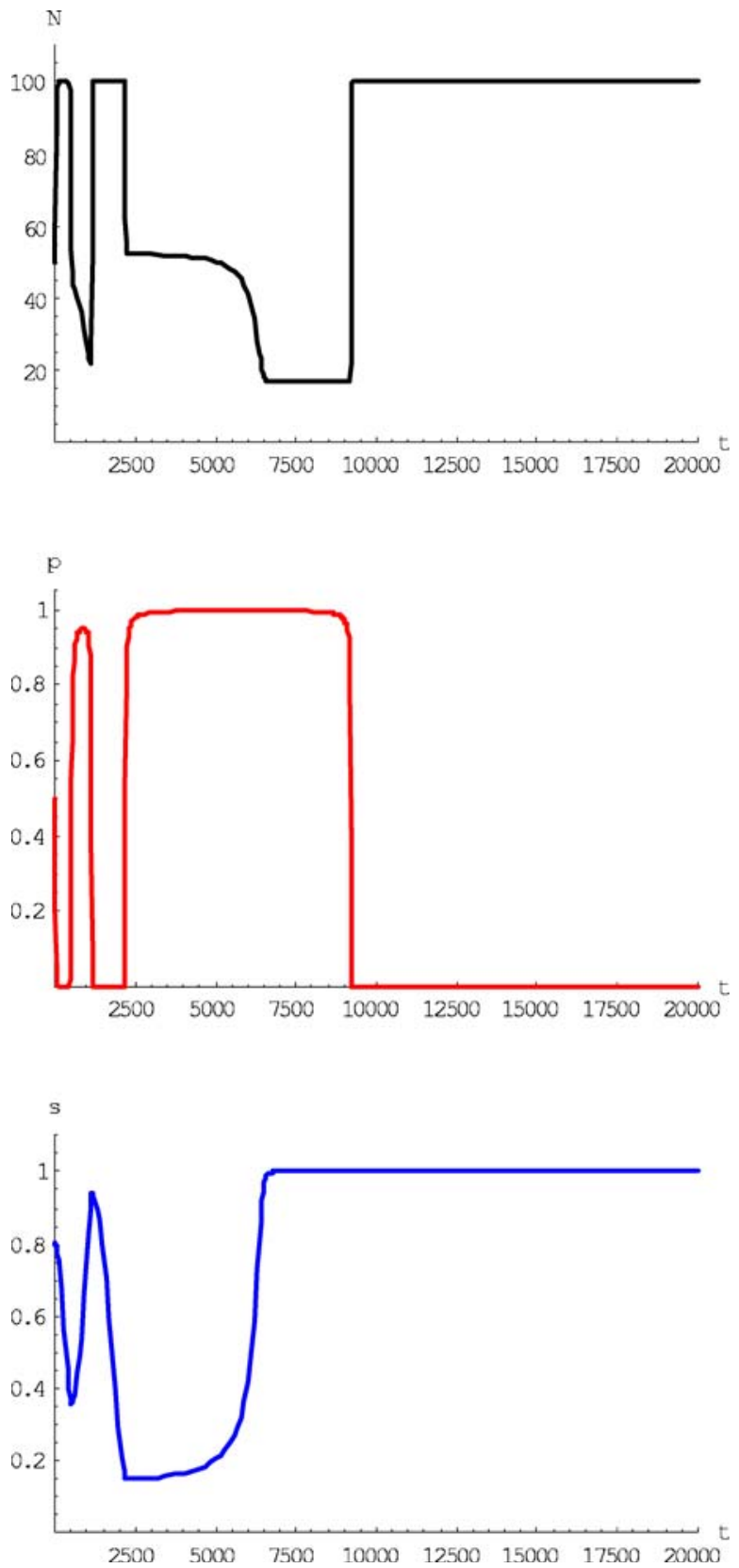

agents have adopted the same strategy, further economic evolution is stopped. Ultimately, $p$ will converge to zero, since $s$ has reached its maximum value, and $N$ reaches $K$.

Remarkably, we did not observe the occurrence of the steady state A. Local stability of A implies that starting close enough to A, the system will 
Fig. 7 Convergence to B with N initial conditions $N_{0}=\bar{N}$, $s_{0}=\bar{s}$, and $p_{0}=p_{E}$
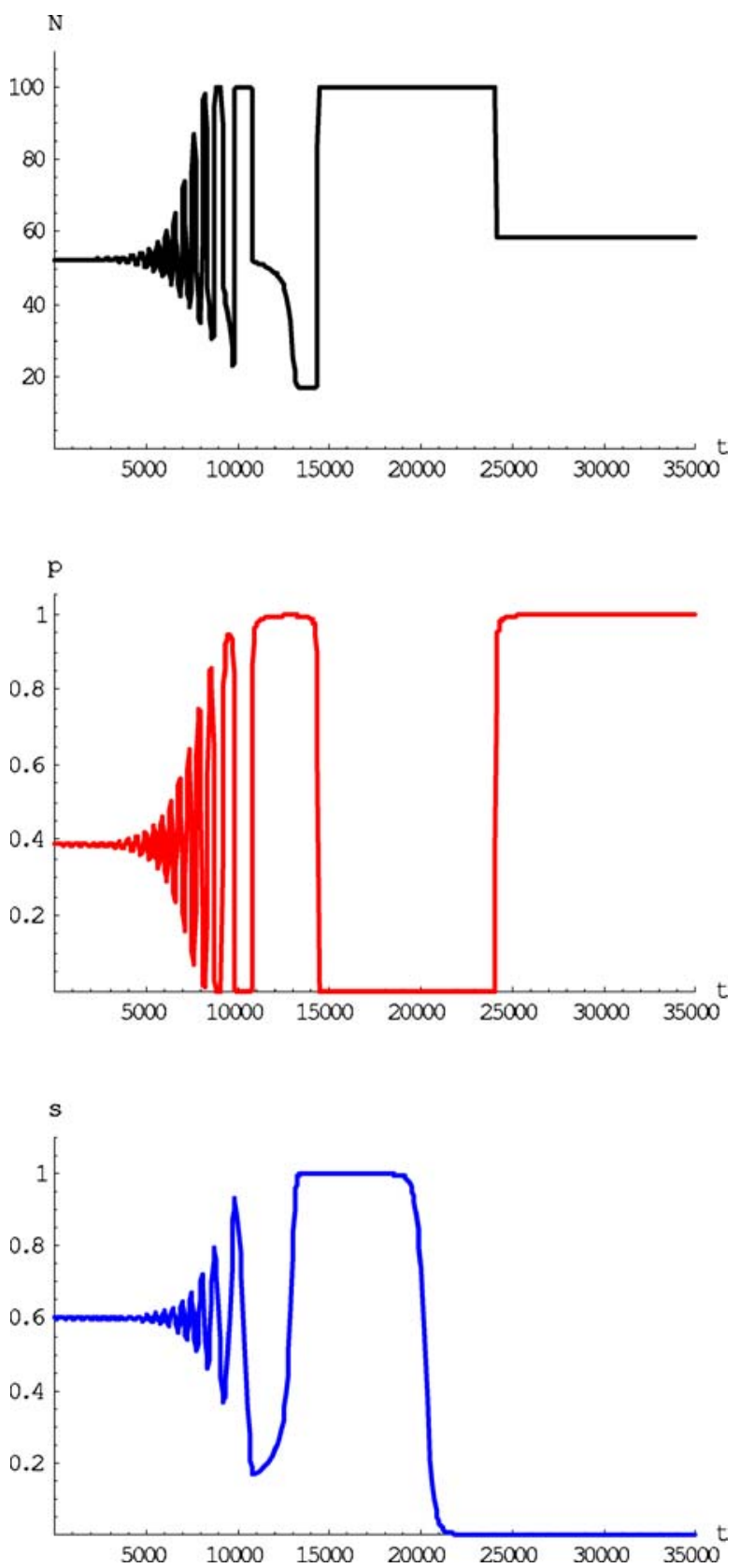

asymptotically converge to it. It can be observed in Fig. 6 that the system is close to A at about $t \simeq 8,000$. Nevertheless, $p$ is not close enough to $p_{A}=1$ for convergence to occur. Further on, $p$ will decrease, since $s=1$, and the system will jump to $\mathrm{C}$. 
Initial conditions also affect the dynamics of the process. Indeed, starting with initial conditions close to the steady state $\mathrm{E}$, the system exhibits more cycles before converging to B. An example is given in Fig. $7 .^{9}$

In Fig. 7, in a first step the gradual motion of $s$ remains close to the threshold $\bar{s}$, and will actually pass beyond it several times. Each of these cycles reproduce the same patterns and phases described in Fig. 3. The cyclical behavior gains amplitude over time, and eventually $s$ approaches zero and the system converges to B.

\section{General implications for policy}

What implications can be drawn from the analysis of the coevolutionary system for agricultural pesticide use policy? When the policy objective is to avoid resistance and reduce the number of pests, convergence to $\mathrm{B}$ is a desirable result for policymakers. Indeed, at B, all pests are susceptible to pesticides and the pest population size remains below the carrying capacity. Furthermore, policy can control the level of $x_{B}$, for instance, by means of pesticide quotas, so as to affect the final level of the pest population. Indeed, a large $x_{B}$ reduces the final level $N_{B}$. However, setting $x_{B}$ too large reduces the threshold $\bar{s}$ below which resistance is reversed. For instance, setting $x_{B}$ such that $W_{A}-m x_{B}=$ $W_{a}$ induces $\bar{s}=0$. In this case, resistance is irreversible and the coevolutionary system will always converge to $\mathrm{D}$, in which $s=0, p=0$, and $N=K$, for any initial level of pesticide use. This underscores the importance of critical ecological thresholds. Policymakers must be aware of these thresholds when implementing policy measures.

Another important result of our analysis is that coevolutionary systems are characterized by nonlinearities and complexity that challenge the task of policymakers. In some cases, the system may converge to a non-desirable state, in which the pest population reaches the carrying capacity, all pests are resistant and all farmers use the intensive strategy, which is costly and, moreover, ineffective. Further, although convergence to such a degenerate state is not frequent in our simulations, precisely identifying the initial conditions leading to $\mathrm{C}$ is problematic, as they are irregularly scattered over the whole set of possible initial values. Therefore, the management of coevolutionary systems is complex and possible irreversible catastrophic scenarios may be caused by the presence of delays between economic and ecological evolution.

An additional result of our analysis deals with the dynamic patterns of the coevolutionary system. Starting with different initial conditions, diverse trajectories are observed before convergence to a steady state. Certain trajectories are characterized by important cyclical patterns. From an economic perspective, there exist trajectories which are more desirable than others in

\footnotetext{
${ }^{9}$ See the logarithmic graph of $p$ in Fig. 10 in Appendix 3.
} 
terms of net present value of total profits. For instance, the net present value of total discounted profits at $T=30,000$ in Fig. 3 is larger than the net present value in Fig. $7 .{ }^{10}$ Numerous fluctuations of profits over time lead to lower total discounted profits over time.

Finally, some general policy implications can be drawn from the study of environment-economy coevolutionary systems. First, the coevolutionary framework sheds light on the speed of selection patterns taking place in the biological and economic worlds. Selection dynamics can occur fast and be characterized by rapid bursts. In addition, biological selection is characterized by rapid accelerations as the frequency of resistant genes become larger. Understanding ecological dynamics and the role of ecological thresholds is of crucial importance for policymakers.

Second, the cyclical behavior observed in our simulations presents some similarities with policies based on rotation of pesticides or based on life cycles of usefulness of pesticides (Georghiou et al.1983; Comins 1984). These policies take into account the possibility that, as soon as the use of a certain pesticide is stopped, the pest population may recover its susceptibility. Such management practices state that once a pesticide becomes ineffective in killing pests, its use must be stopped and possibly replaced by less intensive treatments so that pests can recover susceptibility. Later on, intensive pesticides can again be applied. Similarly, our model promotes alternating between intensive and biological pesticide strategies. Switching to the biological strategy allows for resistance to decline and susceptibility to recovery.

The major innovation of our model is that such alternation in pesticide strategies driven by micro-interactions between the agents is the direct result of changing economic incentives created by ecological evolution. Boundedly rational agents, having information about the pest population size only, have incentives to switch to the biological strategy when the pest population is very large as a result of increasing resistance. Nevertheless, regulation by a central authority can play a great role in influencing some central variables and thus in framing the context of interactions between the agents.

Third and most important, policies in the context of environment-economy coevolution need to make use of the precautionary principle. While so far we assumed that policymakers, unlike the agents, understand the role of biological evolution, this is not necessarily true in reality. A myopic social planner may not be able to foresee the consequences of evolutionary pressures and thus to anticipate the final equilibrium. In such situations, lock-ins into a non-desirable state may be avoided by policies of risk minimizing (Rammel and van den

${ }^{10}$ The net present value of total discounted profits is computed using:

$$
N P V=\int_{0}^{T} m\left(s \pi_{I}+(1-s) \pi_{B}\right) e^{-\delta d t}
$$

with $\delta=0.05$ as the discount rate. Starting with initial conditions as in Fig. 3, we get $N P V=1.07$, while, starting with initial conditions as in Fig. 7, $N P V=0.87$. 
Bergh 2003), which are much in line with the precautionary principle. Such policies involve exploiting the natural environment at a significantly lower level than what is possible, for instance by limiting even further the total level of pesticide use in the system.

\section{Conclusions}

The objective of this chapter has been to combine ecological and economic evolution in a formal model of coevolution applied to the problem of pesticide resistance management in agriculture. The interactions between economic evolution of pesticide strategies, pest population dynamics and genetic evolution of resistance of pests, have been studied in a system of three differential equations.

Given the embedded complexity, the system loses analytical tractability and must be studied using simulation techniques. Numerical illustrations presented some exploratory insights into the dynamics and stability properties of coevolutionary systems. In most of the numerical experiments, a desirable state is achieved in the (very) long run. At that point, there is no resistance in the system, the pest population size lies below the carrying capacity and all agents use the most profitable biological strategy. Further, the analysis sheds light on the types of dynamics observed in such systems, which are characterized by cyclical behavior before convergence to a steady state. Genetic evolution occurs quickly and is characterized by periods of rapid acceleration. In addition, time lags between the different processes can have an impact on the final state reached. Further, the model shows that complexity is an intrinsic element of such systems. Indeed, it is often difficult to identify which initial conditions will lead to an undesirable state. The presence of a few occurrences of degenerate states shows that policymaking in coevolutionary systems is subject to great complexity and possible irreversibilities.

Finally, policy implications have been discussed. A major implication is that policymakers must acknowledge the role of important ecological thresholds. Further, micro-interactions between the agents lead the system to exhibit alternation of pesticide strategies, without the need of governmental intervention. In this context, the role of policymakers consists in varying some critical parameters to frame the complex interactions taking place in coevolutionary systems. Further research is needed to complete the numerical investigation of the system and to study in more detail the effects of the different parameters on the dynamics and stability of the coevolutionary system. Ideally, the model could be tested with some empirics on studies of pesticide resistance management.

Acknowledgements The author thanks Cees Withagen and Jeroen van den Bergh for useful comments and Peter Bolhuis for help with the numerical analysis. This paper was written when the author was affiliated with the VU University Amsterdam, The Netherlands. 


\section{Appendix 1 Solving the three-dimensional coevolution system}

We solve the system:

$$
\left\{\begin{array}{l}
s=0, s=1, N=\bar{N} \\
N=0,\left(p(2-p) r_{A}+r_{a} q^{2}\right)\left(1-\frac{N}{K}\right)=p(2-p) m\left(s x_{I}+(1-s) x_{B}\right) \\
p=0, p=1, s=\bar{s}
\end{array}\right.
$$

Substituting $s=1$ in Eq. 26 gives:

$$
N=\frac{K\left(r_{A} p(2-p)+r_{a} q^{2}\right)-x_{I} K m p(2-p)}{\left(r_{A} p(2-p)+r_{a} q^{2}\right)} .
$$

Substituting for $p=1$ in Eq. 28, we find the coordinates of the steady state A. Similarly, substituting for $s=0$ in Eq. 26, and then for $p=1$ in the resulting equation, gives us the coordinate of the steady state B. Substituting for $p=0$ in Eq. 28, we find the coordinates of the steady states C and D with $s=1$ and $s=0$, respectively. Next, by substituting $N=\frac{a}{b\left(x_{I}+x_{B}\right)}-1$ into Eq. 26, we obtain

$$
\left(r_{A} p(2-p)+r_{a} q^{2}\right)\left(1-\frac{a-b\left(x_{I}+x_{B}\right)}{b K\left(x_{I}+x_{B}\right)}\right)=p(2-p) m\left(s x_{I}+(1-s) x_{B}\right) .
$$

We then substitute Eq. 27 into the above expression:

$$
\left(r_{A} p(2-p)+r_{a} q^{2}\right) \frac{b\left(x_{I}+x_{B}\right)(K+1)-a}{b K\left(x_{I}+x_{B}\right)}=p(2-p)\left(W_{A}-W_{a}\right) .
$$

Observing that $p(2-p)=1-q^{2}$, we get:

$$
r_{A}+\frac{q^{2}}{1-q^{2}} r_{a}=\frac{b K\left(x_{I}+x_{B}\right)\left(W_{A}-W_{a}\right)}{b\left(x_{I}+x_{B}\right)(K+1)-a} .
$$

We must solve the following equation:

$$
\begin{aligned}
{\left[b\left(x_{I}+x_{B}\right)(K+1)-a\right] q^{2} r_{a}=} & b K\left(x_{I}+x_{B}\right)\left(W_{A}-W_{a}\right) \\
& -q^{2} b K\left(x_{1}+x_{2}\right)\left(W_{A}-W_{a}\right) \\
& -r_{A}\left[b\left(x_{I}+x_{B}\right)(K+1)-a\right] \\
& +q^{2} r_{A}\left(b K\left(x_{I}+x_{B}\right)(K+1)-a\right) .
\end{aligned}
$$


Further, for ease of notation we will refer to $u$ and $v$ as:

$$
\begin{aligned}
u & =\left[b\left(x_{I}+x_{B}\right)(K+1)-a\right]\left(r_{a}-r_{A}\right)+b K\left(x_{I}+x_{B}\right)\left(W_{A}-W_{a}\right), \\
v & =b K\left(x_{I}+x_{B}\right)\left(W_{A}-W_{a}\right)-r_{A}\left[b\left(x_{I}+x_{B}\right)(K+1)-a\right],
\end{aligned}
$$

so that we must solve the following equation:

$$
u q^{2}=v
$$

Given that $0 \leq p \leq 1$, there is only one solution:

$$
p=1-\sqrt{\frac{v}{u}}
$$

and combined with $s=\bar{s}$, this gives us the coordinates of the steady state E.

Finally, we obtain the coordinates of the steady state F by setting $N=\bar{N}$ and $p=1$. Equation 26 then becomes:

$$
r_{A} \frac{b\left(x_{I}+x_{B}\right)(K+1)-a}{b K\left(x_{I}+x_{B}\right)}=m\left(s x_{I}+(1-s) x_{B}\right),
$$

and we get

$$
s=\frac{r_{A}\left(b\left(x_{I}+x_{B}\right)(K+1)-a\right)-x_{B} b K m\left(x_{I}+x_{B}\right)}{b K m\left(x_{I}^{2}-x_{B}^{2}\right)} .
$$

\section{Appendix 2 Existence conditions for the steady-states}

- Existence of the steady state A. A necessary condition for the existence of the A-equilibrium is $N_{A} \geq 0$. This holds for:

$$
r_{A} \geq m x_{I}
$$

- Existence of the steady state B. A necessary condition for the existence of the A-equilibrium is $N_{B} \geq 0$. This holds for:

$$
r_{A} \geq m x_{B}
$$


- Existence of the steady states C and D. C and D always exist, since $K>0$.

- Existence of the steady state $E$. $N_{E} \geq 0$ for $a \geq b\left(x_{I}+x_{B}\right)$. Next, the steady state $\mathrm{E}$ exists if $0 \leq p_{E} \leq 1$. This, therefore, requires that the term under the square root must be positive. This requires that $u$ and $v$ must both be positive. This is realized whenever the following inequalities hold:

$$
\begin{aligned}
r_{a}-r_{A} & \leq \frac{b K\left(x_{I}+x_{B}\right)\left(W_{A}-W_{a}\right)}{b\left(x_{I}+x_{B}\right)(K+1)-a} \\
r_{A} & \leq \frac{b K\left(x_{I}+x_{B}\right)\left(W_{A}-W_{a}\right)}{b\left(x_{I}+x_{B}\right)(K+1)-a} .
\end{aligned}
$$

Next, $p_{E} \geq 0$ requires:

$$
\begin{aligned}
1-\sqrt{\frac{u}{v}} & \geq 0 \\
v & \leq u \\
r_{a}\left[b\left(x_{I}+x_{B}\right)(K+1)-a\right] & \geq 0 \\
K & \geq \frac{a}{b\left(x_{I}+x_{B}\right)}-1 .
\end{aligned}
$$

Further, $p_{E} \leq 1$ requires:

$$
\begin{aligned}
1-\sqrt{\frac{u}{v}} & \leq 1 \\
\sqrt{u v} & \geq 0 .
\end{aligned}
$$

This last inequality is always true, so that $p_{E}$ is always inferior to 1 .

The condition $0 \leq s_{E} \leq 1$ is satisfied if:

$$
\begin{aligned}
& W_{A}-W_{a}>m x_{B} \\
& W_{A}-W_{a}<m x_{I} .
\end{aligned}
$$

- Existence conditions of the steady state F. $N_{F} \geq 0$ requires $a \geq b\left(x_{I}+x_{B}\right)$. Next, $0 \leq s_{F} \leq 1$ is satisfied if:

$$
\begin{aligned}
& r_{A} \geq \frac{x_{B} b \operatorname{Km}\left(x_{I}+x_{B}\right)}{b\left(x_{I}+x_{B}\right)(K+1)-a} \\
& r_{A} \leq \frac{x_{I} b K m\left(x_{I}+x_{B}\right)}{b\left(x_{I}+x_{B}\right)(K+1)-a} .
\end{aligned}
$$




\section{Appendix 3 Logarithmic graphs of the evolution of $p$}

Fig. 8 Evolution of $\ln (p)$ in Fig. 3

Fig. 9 Evolution of $\ln (p)$ in Fig. 6

Fig. 10 Evolution of $\ln (p)$ in Fig. 7
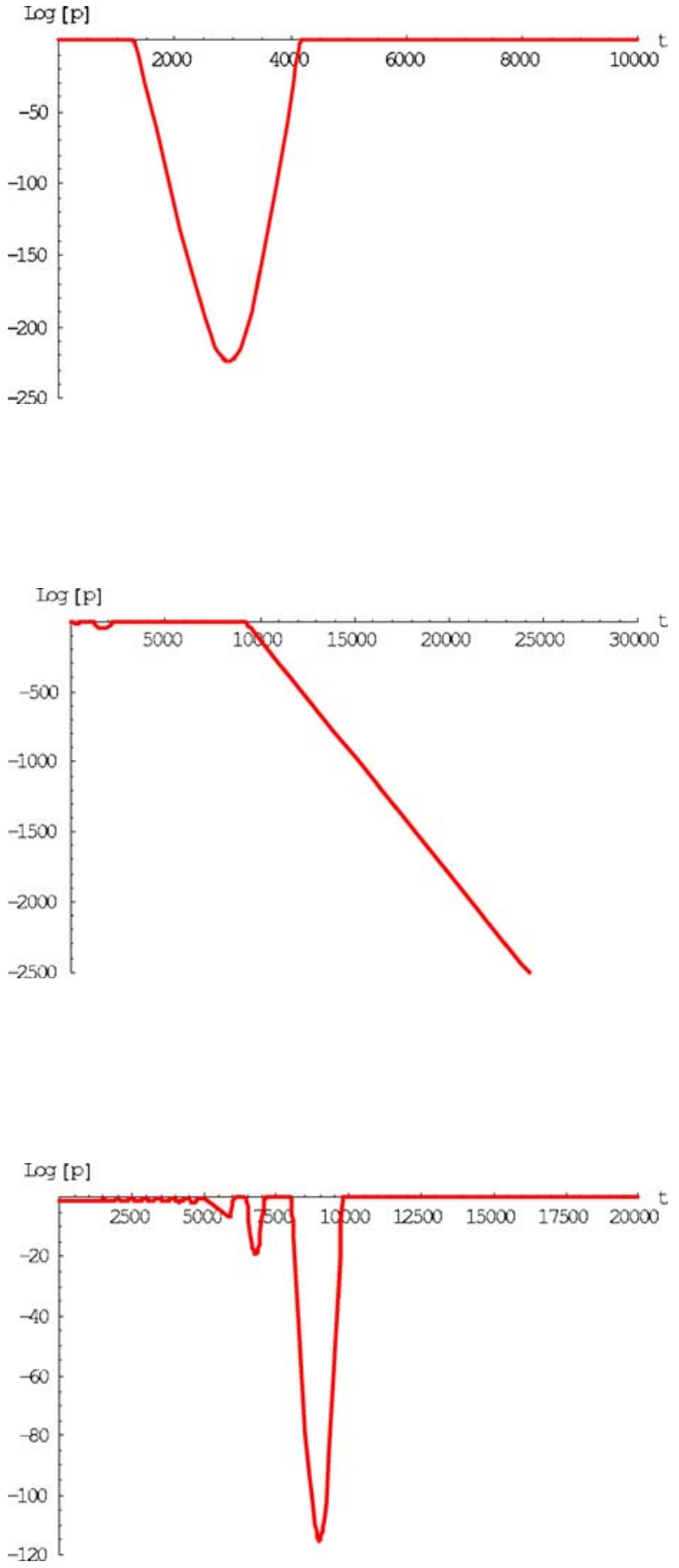


\section{References}

Brown G, Layton D (1996) Resistance economics: social cost and the evolution of antibiotic resistance. Environ Dev Econ 1:349-355

Comins H (1984) The mathematical evaluation of options for managing pesticide resistance. In: Conway G (ed) Pest and pathogen control: strategic, tactical and policy models. Wiley, New York, pp 454-469

David P, Rothwell G (1996) Standardization, diversity and learning: strategies for the coevolution of technology and industrial capacity. Int J Ind Organ 14(2):181-201

Dieckmann U, Law R (1996) The dynamical theory of coevolution: a derivation from stochastic ecological processes. J Math Biol 34:579-612

Dieckmann U, Marrow P, Law R (1995) Evolutionary cycling in predator-prey interactions: population dynamics and the Red Queen. J Theor Biol 176:91-102

Ehrlich P, Raven P (1964) Butterflies and plants: a study in coevolution. Evolution 18:586-608

Georghiou G (1980) Insecticide resistance and prospects for its management. Residue Rev 76: $131-145$

Georghiou G, Lagunes A, Baker J (1983) Effects of insecticide rotations on evolution of resistance. In: Miyamoto J (ed) IUPAC pesticide chemistry, human welfare and the environment. Pergamon, Oxford, pp 183-189

Heino M (1997) Management of evolving fish stocks. IIASA Working paper IR-97-062. Laxenburg, Austria

Herre E (1989) Coevolution of reproductive characteristics in twelve species of new world figs and their pollineator wasps. Experientia 45:637-647

Hilborn R (1994) Chaos and nonlinear dynamics. Oxford University Press, Oxford, New York

Hooper L, Gordon J (2001) Commensal host-bacterial relationship in the gut. Science 292: 1115-1118

Laxminarayan R (2001) Economics of antibiotic resistance: a theory of optimal use. J Environ Econ Manage 42(2):183-206

Levin S, Udovic G (1977) A mathematical model of coevolving populations. Am Nat 111:657-675

Lumsden C, Wilson E (1981) Genes, mind and culture. the coevolutionary process. Harvard University Press, Cambridge, MA

May R, Anderson R (1983) Epidemiology and genetics in the coevolution of parasites and hosts. Proc R Soc Lond B 219:281-313

Munro A (1997) Economics and biological evolution. Environ Resour Econ 9:429-449

National Research Council (1986) Pesticide resistance: strategies and tactics for management. National Academy Press, Washington, DC

Norgaard R (1984) Coevolutionary development potential. Land Econ 60:160-173

Norgaard R (1994) The coevolution of economic and environmental systems and the emergence of unsustainability. In: England R, Arbor A (eds) Evolutionary concepts in contemporary economics. University of Michigan Press, Ann Arbor, MI

Policansky D (1993) Fishing as a cause of evolution of fishes. In: Stokes T, McGlade J, Law R (eds) The exploitation of evolving resources. Springer, Berlin, pp 1-18

Rammel C, van den Bergh J (2003) Evolutionary policies for sustainable development: adaptive flexibility and risk minimizing. Ecol Econ 47:121-133

Regev U, Shalit H, Gutierrez A (1983) On the optimal allocation of pesticides with increasing resistance: the case of Alfalfa Weevil. J Environ Econ Manage 10:86-100

Roughgarden J (1979) Theory of population genetics and evolutionary ecology: an introduction. MacMillan, New York

Schneider S, Londer R (1984) Coevolution of climate and life. Sierra Club Books, San Francisco

Stokes T, McGlade J, Law R (1993) The exploitation of evolving resources. Springer, Berlin

Strogatz S (1994) Nonlinear dynamics and chaos. Addison-Wesley, Reading, MA

Tyler Miller G (1992) Environmental science. Wadsworth, Belmont, CA

van den Bergh J, Stagl S (2003) Coevolution of economic behaviour and institutions: towards a positive theory of institutional change. J Evol Econ 13(3):289-317 